\title{
Widely tunable cavity-enhanced ultrafast spectroscopy
}

\author{
Myles C. Silfies ${ }^{1}$, Yuning Chen ${ }^{1}$, Henry Timmers ${ }^{2}$, Abijith S. Kowligy ${ }^{2}$, Alex Lind ${ }^{2,3}$, Scott \\ A. Diddams ${ }^{2,3}$, and Thomas K. Allison ${ }^{1, *}$ \\ ${ }^{1}$ Stony Brook University, Stony Brook, New York 11794, USA \\ ${ }^{2}$ National Institute of Standards and Technology, Boulder, Colorado 80305, USA \\ ${ }^{3}$ University of Colorado, Boulder, Colorado 80309, USA
}

\begin{abstract}
Generation of widely tunable frequency combs in the UV, visible, and infrared is discussed for use in cavity-enhanced transient absorption spectroscopy on gas-phase clusters. Progress towards cavityenhanced two-dimensional spectroscopy is also presented.
\end{abstract}

\section{Introduction}

Typically ultrafast optical spectroscopy is done on optically dense samples such as solids or liquids which have relatively large absorption of both pump and probe pulses. Unfortunately, there are many samples of interest, such as gas-phase clusters, which only exist at densities lower than those which are easily studied with traditional methods. In order to study vibrational dynamics of these low-density samples the interaction strength must be increased; either by extending the physical size of the sample or resonantly enhancing the signal. The former is not feasible for gas-phase clusters in a molecular beam. The latter option must therefore be utilized by borrowing techniques from the already successful fields in cavity-enhanced linear spectroscopy [1] and combining them with ultrafast methods.

In order to resonantly enhance the signal, a frequency comb laser is coupled to a ring cavity constructed around the sample. The effective sample interaction length, and therefore signal, is then proportional to the cavity finesse. By also resonantly enhancing the pump light using a geometry shown in figure $1 \mathrm{~b}$ ), the excitation is also enhanced. We have previously shown that a sensitivity of $\Delta \mathrm{OD}=1 \times 10^{*} / \sqrt{\mathrm{Hz}}$ can be achieved in a transient absorption measurement of gas-phase molecular iodine [2]. This demonstration experiment was done with both the pump and probe light at $529 \mathrm{~nm}$. However, in order to have a more widely applicable spectrometer, the pump and probe wavelengths must be tunable. Experiments currently under development require tunability across the ultraviolet (UV), visible, and mid-infrared (MIR) spectral regions.

\footnotetext{
* Corresponding author: thomas.allison@stonybrook.edu
} 


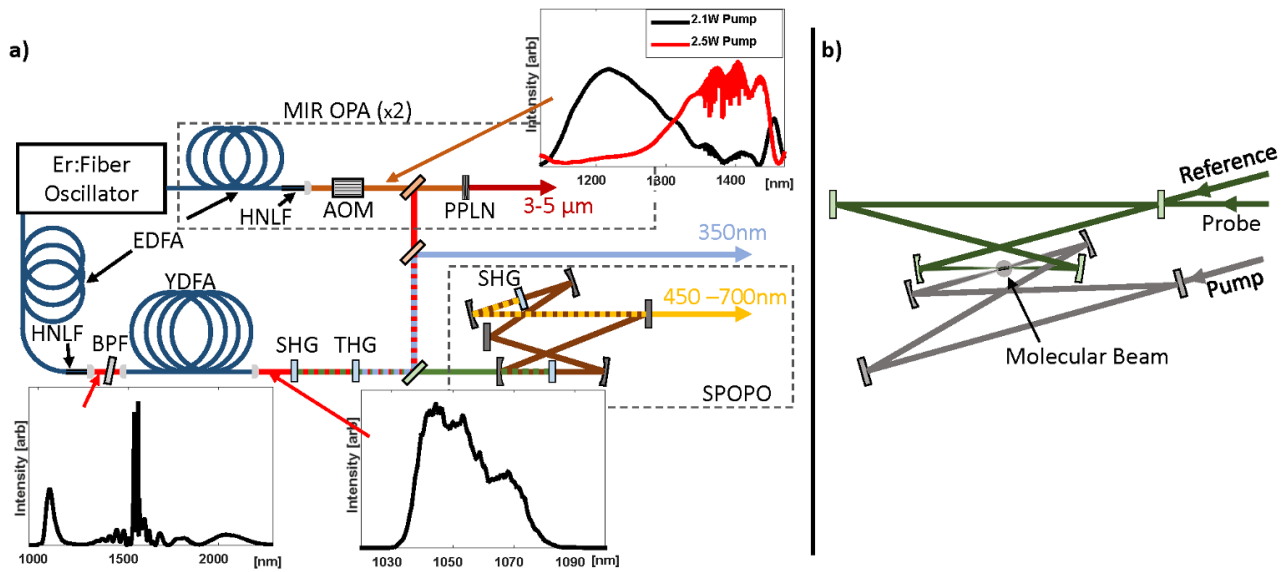

Fig. 1. a.) Layout for the generation of tunable optical frequency combs in UV, visible, and MIR spectral regions. Typical spectra are included for HNLF output, amplified $1050 \mathrm{~nm}$ pump, and tunable near-infrared signal combs. b.) Experimental cavity layout, a gas slit nozzle is used to generate a molecular beam at the foci of two crossed ring cavities for the enhancement of both pump and probe light. Reference cavity is used for noise subtraction [2].

\section{Generation of tunable frequency combs}

The layout for tunable frequency comb generation is depicted in figure 1a). The initial comb is generated using an erbium fiber oscillator centered at $1550 \mathrm{~nm}$ with a repetition rate of $100 \mathrm{MHz}$. To act as high-power pump for further nonlinear processes, one output of the oscillator is amplified in a nonlinear erbium doped fiber amplifier (EDFA) and compressed in a standard fiber patch cord to a duration less than 50 fs due to the fiber's inherit anomalous dispersion at $1550 \mathrm{~nm}$. This pulse is then directly coupled into a short $(<5 \mathrm{~cm})$ piece of highly nonlinear fiber (HNLF) which generates an optical soliton and a phase-matched dispersive wave [3]. Fiber lengths and pump diode powers are tuned to center the dispersive wave at $1050 \mathrm{~nm}$ with an average power of approximately $30 \mathrm{~mW}$. This portion of the spectrum is then selected by a band pass filter (BPF) and used to seed a two-stage ytterbium doped fiber amplifier (YDFA) consisting of a core-pumped preamplifier, fiber-based stretcher, cladding pumped power amplifier, and a grating compressor to generate a $10 \mathrm{~W}$ average power comb with a pulse duration less than $100 \mathrm{fs}$ as described in [4]. We have measured the output comb tooth linewidth to be less than 10 $\mathrm{kHz}$.

For the generation of ultraviolet and visible frequency combs, the output of the YDFA is first frequency doubled via a second harmonic generation (SHG) crystal and frequency tripled in a sum-frequency third harmonic generation (THG) crystal. The third harmonic is directly used as the UV pump for ultrafast experiments. The second harmonic is used as a pump in a synchronously pumped optical parametric oscillator (SPOPO), shown at the bottom of figure 1a), which consists of a dual focus cavity that is matched to the oscillator cavity length and has a difference frequency generation (DFG) crystal at the first focus to generate a signal and idler pair tunable from 900 to $1400 \mathrm{~nm}$. An SHG crystal at the second cavity focus frequency doubles either the signal or the idler to generate a tunable visible frequency comb from 450 to $700 \mathrm{~nm}$. Both the UV and visible combs are several hundred $\mathrm{mW}$ average power and approximately $200 \mathrm{fs}$ in duration before being coupled into the pump and probe enhancement cavities. The probe cavity is made from matched pairs of positively and negatively chirped mirrors for a round trip GDD of less than $100 \mathrm{fs}^{2}$ across the entire tuning range of the visible comb to maintain a short pulse duration at the sample. 
MIR comb generation is done in a single-pass optical parametric amplifier (OPA), shown at the top of figure 1a), which is made up of a periodically poled lithium niobate (PPLN) crystal pumped by the YDFA output already discussed. The desired signal wavelength for difference frequency generation (DFG) is produced by a second EDFA and HNLF branch similar to the one used for the YDFA seed but optimized for output centered between 1300 and $1500 \mathrm{~nm}$ for an idler output tunable from 3 to $5 \mu \mathrm{m}$ [5]. An acousto-optic modulator (AOM) is placed in the signal arm in order to tune the carrier offset frequency of the generated idler comb to match the offset of the enhancement cavity. Several planned experiments will require a single MIR comb as a probe with a UV pump to study the vibrational dynamics of excited gas-phase molecules and clusters. A third EDFA, HNLF, and OPA will also be implemented for use in cavity-enhanced two-dimensional MIR spectroscopy as described in [6]. GDD optimized broadband cavity mirrors for the MIR combs have also been designed.

\section{References}

1. G. Gagliardi, H.-P. Loock, eds., Cavity Enhanced Spectroscopy and Sensing, (Springer, 2013)

2. M. A. R. Reber, Y. Chen, T. K. Allison, Optica 3, 311 (2016)

3. D. Brida, G. Krauss, A. Sell, A. Leitenstorfer, Laser Photonics Rev. 8, 409 (2014)

4. X. L. Li, M. A. R. Reber, C. Corder, Y. Chen, P. Zhao, T. K. Allison, Rev. Sci. Inst. 87, 093114 (2016)

5. D. L. Maser, G. Ycas W. I. Depetri, F. C. Cruz, S. A. Diddams, Appl. Phys. B 123, 142 (2017)

6. T. K. Allison, J. Phys. B: At. Mol. Opt. Phys. 50, 044004 (2017) 